\title{
A step by step process for graduate nursing students seeking best evidence on palliative care
}

\author{
Elizabeth Bonham ${ }^{1 *}$, Erin L Federspiel ${ }^{2}$, Benita Randolph ${ }^{2}$ and Dustie L Barnett ${ }^{2}$ \\ ${ }^{1}$ Department of Nursing, University of Southern Indiana, USA \\ ${ }^{2}$ Master of Science in Nursing Program, University of Southern Indiana, USA
}

\begin{abstract}
The complex healthcare system today requires that nurses apply the best evidence for desired patient outcomes that improve quality of life. One way to do so is to acquire a skill set of literature review that the nurse can translate into best practices. Learning an evidence-based practice technique assures application of the ability to locate and utilize scientific evidence. In this article, a step by step process of finding the evidence is presented as taught in a graduate nursing program. Learning the process as students in a Master of Nursing Science program ensures that the future Advanced Practice Registered Nurse discovers evidence that substantiates best practices. The six-step process includes forming a clinical question, selecting a clinical guideline, identifying databases, creating search terms and key words, choosing particular evidence, and summarizing the evidence. Searching the evidence through use of the step by step process to answer a clinical question regarding palliative care is presented as a case study.
\end{abstract}

\section{A step by step process}

The current health care system with its rapid pace, complex comorbidities, and multiple providers requires that nurses be able to utilize the best practice for patient care. Knowing the most effective procedures calls for a system of evidentiary retrieval in a systematic manner. The effective procedures also necessitate an appraisal method of evidence that contributes to efficacious care of a patient population. Melnyk and Fineout-Overholt's publication is useful in teaching the process and components of selecting evidence that translates to patient care outcomes [1]. Evidence based care has been taught in both nursing and medical schools for some time [2-5]; however, there remains a cadre of practitioners who are not aware of the process and may continue care based on the "we have always done it this way" refrain. Teaching the beginning Master of Nursing Science (MSN) student the evidence-based process continues to elevate the use of science for best practices. The purpose of this paper is to describe the steps used by graduate nursing students to find the best evidence for their defined clinical topic of palliative care. A case study is included to illuminate the process.

\section{Evidence based care}

One objective of the graduate nursing course in the authors' university MSN program is that the student will be able to integrate theory, evidence, clinical judgment, clinical guidelines, research, and interprofessional perspectives in order to improve practice as well as be able to critically appraise the evidence for practice decisions. To achieve this outcome, students are first taught about the evidence-based practice care (EBP) process which begins with discerning the clinical question. In doing so, the nurse identifies the patient population, an intervention or interest, a comparison intervention or status, and the outcome. This information can be operationalized as the PICOT question. The second step is to search for the best evidence. Doing so requires library skills in searching databases, defining key search terms, and maintaining well defined and preset criteria. The third step employs a critical appraisal of the selected literature (evidence) that answers what the study results were, their validity, and if the results will facilitate patient care. Integrating the evidence, the fourth step of the EBP process, takes into consideration the healthcare provider's expertise, the clinical assessment of the patient, the healthcare resources, patient preferences and values as well as the patient's participation in clinical decision making. Importantly, in order to evaluate the desired outcome, the fifth step analyzes if the treatment worked, how effective the treatment was and what the outcomes are.

There are several techniques students learn in order to find the evidence. The first technique is to locate a clinical practice guideline that relates to the PICOT question. A clinical practice guideline initiates specific diagnostic and treatment regimens that have been reviewed by practitioners in a rigorous systematic review. The statement is usually issued by a third party and offers recommendations for specific care that providers may consider. Next, the students identify databases that are used to find the literature as well as describe the search terms and the step by step process used in the inclusion and elimination of search terms and studies. The final technique is to select the literature that can be used to answer the clinical question. The literature must address the concepts in the PICOT question, be research articles, and published within the last 5-7 years. Table 1 summarizes the step by step process that is described in this article.

*Correspondence to: Elizabeth Bonham, 8600 University Boulevard, Evansville, IN 47712, Tel: 812-465-1164, E-mail: ecbonham@usi.edu

Key words: evidence-based practice, clinical guideline, search terms, databases, palliative care

Received: June 18, 2018; Accepted: July 12, 2018; Published: July 19, 2018 
Table 1. Step by step process of finding the evidence

\begin{tabular}{|c|c|}
\hline 1 & $\begin{array}{l}\text { Develop the clinical question known as the PICOT question that identifies } \\
\text { the population of interest, intervention, comparison, and outcome }\end{array}$ \\
\hline 2 & $\begin{array}{l}\text { Identify a clinical practice guideline that relates to the PICOT question } \\
\text { topic. }\end{array}$ \\
\hline 3 & Identify databases that can be used to find literature. \\
\hline 4 & $\begin{array}{l}\text { Develop search terms and describe the process of inclusion and } \\
\text { elimination } \mathrm{f} \text { key words. }\end{array}$ \\
\hline 5 & Select the evidence, appraise the literature using a level of hierarchy. \\
\hline 6 & $\begin{array}{l}\text { Provide a conclusion about the evidence that can be translated to changed } \\
\text { practice in patient care. }\end{array}$ \\
\hline
\end{tabular}

\section{Palliative care}

In this graduate nursing course, students select from five clinical topics identified by faculty - hypertension, cardiovascular disease prevention in women, diabetes management, mental health care, and palliative care (PC). In view of the complexities of patient care and comorbidities found in many patients in intensive care units, the study of palliative care is increasingly studied by students as they search for ways to improve quality and quantity of life as well as making meaning of patient lives. In the United States, palliative care is used sporadically and with some genuine misunderstanding of its use in enhancing quality of life. The World Health Organization defines PC as an approach that may optimize quality of life for patients with a life limiting illness and their family members [6,7]. Aldridge and colleagues note that because there are more people with life threatening and end stage diseases and the notion of caring for people with non-curative diseases causes challenges for families, palliative care offers substantial benefits. For the purpose of this article, palliative care was the selected clinical topic.

\section{PICOT question}

An important part of the research process is the formulation of the PICOT question. This provides the foundation for the research and clarifies for the reader and the research team what is being studied. The PICOT must be an answerable question with relevant supporting data. In order to be a quality research question, the PICOT must be achievable, thought provoking, unique, and ethical [8].

In formulating this question, the researchers will first identify population $(\mathrm{P})$, which could include among others, people of similar ages, illnesses, and ethnicity. The population tenet requires the individuals being studied share a common factor that pertains directly to the research. The population study of the PICOT also often identifies the problem being addressed [9]. The intervention (I) component is the treatment or action that the population will or will not receive. This should be founded on evidence-based practice. Interventions can be based on recent research or patient observation as well as looking at current facility practice to determine what can be done in order to improve patient care. Researchers implement the intervention in order to establish new best care practices based on the efficacy of the intervention [9]. The control for the study is found in the comparison (C) section. These are the individuals or groups of individuals that will be used as a reference point and will not be receiving the intervention, only the standard protocol that is already in place [8]. The part of the PICOT where the researchers can recognize the effect of the intervention is in the outcomes $(\mathrm{O})$. These are the measurable results the investigators will evaluate to determine whether or not the intervention was effective. This is the period in which pertinent statistics will be obtained. The information gathered throughout the study will be compiled in order to decide whether or not the intervention has a positive effect on patient care.
The PICOT question is essential to a research study. It presents the researchers with parameters in which to conduct a literature search and includes relevant terms for searching databases. It has been shown that clinicians, specifically in the palliative care field, are somewhat ineffective at conducting searches. By using databases in an unproductive manner, the searches can yield an overwhelming number of results or eliminate the most relevant studies [10]. By identifying specific demographics and illnesses in a group of people, the investigator will have a clear direction when beginning the search for data.

\section{Clinical guidelines}

Clinical guidelines are "statements that include recommendations intended to optimize patient care that are informed by a systematic review of evidence and an assessment of the benefits and harms of alternative care options" [11]. These guidelines provide parameters and direction for performing best care practices for a specific population or community of patients. These can be very helpful as they are a compilation of the most recent research regarding the subject, thus saving the researcher time investigating these elements on their own.

Choosing a clinical guideline that relates to the area of interest is the next step in the research process after a PICOT question has been formed. In order to find a relevant guideline, the researcher will search for the terms found in the PICOT question. For example, one of the authors conducted a search in this manner on the subject of massage as an adjunctive therapy to palliative care in cancer patients. The search was performed on the PubMed database by using the terms palliative care, alternative therapy, and cancer in conjunction with one another. This search returned several guidelines that met the search criteria and authors were then tasked with finding the one most applicable to the PICOT question.

To determine if a clinical guideline is relevant to a research study, one can use the AGREE II method, which stands for Appraisal of Guidelines, Research, and Evaluation. This method includes 23 elements that are divided between six domains. The domains include scope and purpose, stakeholder involvement, rigor of development, clarity of presentation, applicability, and editorial independence [12] This assessment has proven effective in creating as well as evaluating different clinical guidelines. The AGREE II method is user-friendly, even to researchers at a beginner level [12]. By using this strategy, the researchers can easily identify what guideline would be most appropriate for their study regarding cancer patients, massage, and palliative care. The first two steps of finding the evidence have been described in this section.

\section{Selecting a database}

Selecting a database is the third step in the process of searching for the best evidence. A database is the storage of organized digital data controlled by a database management system software for retrieval of specific information. Databases provide rich information of scholarly, peer reviewed, updated, and empirical articles. EBSCOHost is a databank that holds numerous databases catered to different disciplines [13]. Facchiano and Snyder state that out of the library and web-based databases, the library databases are more reliable [14].

It is necessary to select the most appropriate database before searching for articles in order to avoid unnecessary and irrelevant information. It is possible to become inundated with articles if precision is not used in choosing a database. Richter and Austin state that while the Physiotherapy Evidence Database can be used specifically for 
physical therapy, another database such as Cumulative Index of Nursing and Allied Health Literature (CINAHL) applies to a much wider degree of medical and health related information [15]. Facchiano and Snyder affirm this by stating that the selection process of a meaningful source can be intimidating because of the considerable number of available electronic databases and their unique search protocol. Therefore, the database(s) selected must be considered carefully and should coincide with the PICOT question [9].

The methodology of searching for articles to answer the clinical question can be simplified by selecting multiple databases for a single search [13]. For example, databases such as MEDLINE and CINAHL can be chosen as the selected databases of interest before the search begins on EBSCOHost. The search process can be further clarified by limiting searched articles to full text, abstract only, date of publication, and source types [14]. This decreases the amount of wasted time and effort of painstakingly searching through significant amounts of data. For example, for the PICOT question, "In chronically ill patients admitted to an intensive care unit (ICU), how does patient communication with RNs/APRNs who are formally educated in palliative care (PC) compare to patient communication with RNs/ APRNs lacking formal education in PC affect perceived level of comfort during hospitalization?", ProQuest Health, Medline with full text, and CINAHL Plus with full text were utilized. The parameters for the search were set to publications from 2012 to 2017, the English language, and academic journals to conduct a more refined search.

\section{Selecting key search terms}

The fourth step in the process of searching for the best evidencebased practice is selecting key search terms. The vocabulary used for the search is called the term or the word. Depending on what is desired terms can be specific or complex. The complexity of the term will yield wider amounts of information whereas specificity will yield narrower results. Terms or phrases applicable to the topic of interest or the PICOT question are called keywords or natural language. Key search terms are specific to avoid a vast number of irrelevant articles and to fully represent the topic of interest [14]. In the previous PICOT question example, the search terms palliative care, patient satisfaction, education, nursing, United States, palliative care nursing, palliative care education, medical, critical care, barriers and physicians were used. A maximum of three search terms were used at one time which yielded various results.

Another search term strategy is the use of Medical Subject Headings $(\mathrm{MeSH})$ terms which are also called controlled vocabulary [14]. MeSH terms are related to the key terms used. In other words, this strategy provides synonyms used to run the search along with the keywords or key search terms [15]. MeSH is activated by simply selecting the "related word" option or "map term to subject heading" in the advance search. The use of MeSH terms has been said to improve clarity and consistency and increase search effectiveness [15]. For example, using the MeSH strategy with the keyword 'chronically ill' yields articles with terms such as debility, frailty, and infirmity. Another example of the $\mathrm{MeSH}$ strategy using the keyword 'palliative care' delivers articles with the terms 'hospice care' and 'end of life care. Regardless of the search strategy used, preliminary search terms should be created. After trial and error, the search terms that are not yielding the desired results may be eliminated.

\section{Selecting the evidence}

The fifth step involves selecting the evidence. After the desired articles are found, the best evidence to guide the PICOT question must be selected carefully. One way of finding the best evidence is by the notion of strength. The strength of an evidence is a way to classify evidence-based care on scientific precision and quality. The strength of an article is ranked into levels of evidence [16]. There are numerous levels of evidence hierarchies with some ranging from levels I to V and others from I to VII. To clarify a particular nursing organization's level of evidence hierarchy, Hawks utilized the level of evidence from Polit and Beck's publication of Nursing Research [17-18]. Level I is the strongest level of evidence and consists of systematic reviews, metaanalyses, and clinical practice guidelines of randomized controlled trials (RCTs). These articles fall in the range of experimental studies. Level II evidence covers a single RCT or a systematic review of RCT and quasi experimental studies. Level III is a well-designed controlled trial without randomization. Level III articles are considered nonexperimental studies. Level IV is evidence from case-controlled and cohort studies. Level V is a systematic review of several descriptive and/ or qualitative studies. Level VI is evidence from a single descriptive or qualitative study, and finally, Level VII is evidence from the opinion of authorities and/or reports of expert committees [17].

After the level of evidence is identified for each article, it is advisable that a rapid appraisal be completed. To appraise an article is to evaluate the evidence for weaknesses, strengths, and value in relation to the ability to answer or provide the needed information for the PICOT question. Relevance/significance, validity, reliability, applicability, clarity, precision, completeness, and accuracy are some of the areas that are appraised [19]. For the novice researcher, appraising has been made easier with the availability of various rapid appraisal tools or templates [16]. Because of the study design differences, the appraisal tools will differ as well. For example, the appraisal tool for a qualitative study will differ for a quantitative study. Looking once again at the earlier clinical question, "In chronically ill patients admitted to an intensive care unit (ICU), how does patient communication with RNs/APRNs who are formally educated in palliative care (PC) compare to patient communication with RNs/APRNs lacking formal education in PC affect perceived level of comfort during hospitalization?", each article selected was appraised with a research design specific template that addressed the concepts of the PICOT question. After completing the appraisal, an evaluation table (see Table 2) may be helpful to complete the evaluation process.

A case study will be described next to elucidate the steps above in finding the best evidence.

\section{Case study}

For the purpose of this article, the graduate nursing students seeking the best evidence on palliative care formulated this PICOT question: In adult oncology patients ages 18 and older, how does massage therapy compared to standard care affect pain relief and anxiety after one or more massage therapy sessions averaging 15 to 30 minutes each?

The evidence based clinical practice guideline chosen, Complementary therapies and integrative medicine in lung cancer: Diagnosis and management of lung cancer, relates directly to the PICOT

Table 2. Evaluation Table

\begin{tabular}{|c|c|c|c|c|c|c|c|}
\hline $\begin{array}{c}\text { Student Name/ } \\
\text { Citation }\end{array}$ & Framework & $\begin{array}{c}\text { Design/ } \\
\text { Method }\end{array}$ & $\begin{array}{c}\text { Sample/ } \\
\text { Setting }\end{array}$ & Variables & Measurement & Data Analysis & Findings \\
\hline
\end{tabular}


question presented and supports the need to integrate complementary therapies with standard care therapies. In lung cancer patients experiencing pain and anxiety, mind-body modalities such as massage therapy are recommended as an adjunct treatment to improve quality of life [20].

The search for literature related to the PICOT question was performed using the databases CINAHL, Cochrane Library, Medline, and ProQuest. The search process was further limited to full text, date of publication, and source types in order to obtain scholarly and/or peer-reviewed sources, and the most complete list of relevant citations.

Keyword searches began using a specific combination of words from the PICOT question such as adult cancer patients, massage therapy, pain relief, and anxiety. A maximum of three search terms were used at one time to enhance the selection of articles received. Variations of the search terms were used at one time to acquire the most suitable articles. Articles were reviewed for relevance to the PICOT question. This process continued until suitable articles were discovered.

The articles selected were based on the strength of the evidence, level I, the strongest level. These included clinical practice guidelines of RCT's and systematic reviews. A rapid critical appraisal was then completed for each article using a research design specific template. With the selected literature, progression of the next step of summarizing the literature and completing the evaluation table can now be taken in the venture of answering the PICOT question.

\section{Conclusion}

Contemporary health care requires that in order to deliver the best care, the advanced practice nurse acquire a toolbox of skills that includes searching the literature for best practices. This skillset is one that is taught in graduate school and is helpful in many ways. The process of learning how to find the evidence has been described in this article. Appraising the evidence is also a skillset that can translate into better care. As knowledge evolves, nurses must be able to accrue new areas of expertise. Becoming proficient with literature searches and translating the evidence based on a selected level of evidence aids the nurse to provide patient centered care that is both scientific and valued by the patient.

\section{References}

1. Melnyk BM, Fineout-Overholt E (2015) Evidence-based practice in nursing and healthcare. 3rd ed. Philadelphia: Lippincott Williams \& Wilkins.

2. Fineout-Overholt E, Levin RF, Melnyk BM (2004) Strategies for advancing evidence-based practice in clinical settings. J New York St Nurse State Nurses Assoc 35: 28-32. [Crossref]
3. Stetler CB (2001) Updating the Stetler model of research utilization to facilitate evidence-based practice. Nursing Outlook 49: 272-279. [Crossref]

4. Stevens KR (2001) An introduction to evidence-based practice. Newborn \& Infant Nursing Review 1: 6-10.

5. Sackett DL, Rosenberg WM, Gray JA, Haynes RB, Richardson WS (1996) Evidence based medicine: What it is and what it isn't - It's about integrating individual clinical expertise and the best external evidence. Brit Med J 312: 71-72. [Crossref]

6. World Health Organization (2018) Cancer: Palliative care. World Health Organization.

7. Aldridge MD, Hasselaar J, Garralda E, Van der Eerde M, Stevenson D, et al. (2016) Education, implementation, and policy barriers to greater integration of palliative care: A literature review. Palliative Med 30: 224-239. [Crossref]

8. Riva J, Malik K, Burnie S, Endicot A, Busse J (2012) What is your research question? An introduction to the PICOT format for clinicians. J Can Chiropr Assoc 56: 167-171. [Crossref]

9. Facchiano L, Snyder CH (2012a) Evidence-based practice for the busy nurse practitioner: Part one: Relevance to clinical practice and clinical inquiry process. $J \mathrm{Am}$ Acad Nurse Prac 24: 579-586. [Crossref]

10. Damarell RA, Tieman JJ (2015) Searching PubMed for a broad subject area: How effective are palliative care clinicians in finding the evidence in their field? Health Info Libr J 33: 49-60. [Crossref]

11. Institute of Medicine $[\mathrm{IOM}]$ (2011) Clinical Practice Guidelines We Can Trust Washington, DC: The National Academies Press. [Crossref]

12. Brouwers MC, Kho ME, Browman GP, Burgers JS, Cluzeau F, et al. (2010) AGREE II: Advancing guideline development, reporting and evaluation in health care. Can Med Assoc J 182: E839-E842. [Crossref]

13. Dillard D (2014) Selecting EBSCOHost databases and applying them to a life care planning search, part I. Journal of Nurse Life Care Planning 14: 59-64.

14. Facchiano L, Snyder CH (2012b) Evidence-based practice for the busy nurse practitioner: Part two: Searching for the best evidence to clinical inquiries. $J$ Am Acad Nurse Prac 24:640-648. [Crossref]

15. Richter RR, Austin TM (2012) Using MeSH (Medical Subject Headings) to enhance PubMed search strategies for evidence-based practice in physical therapy. Phys Ther 92: 124-132. [Crossref]

16. Facchiano L, Snyder CH (2012c) Evidence-based practice for the busy nurse practitioner: Part three: Critical appraisal process. J Am Acad Nurse Prac 24: 704-715. [Crossref]

17. Hawks JH (2016) Changing the level of evidence. Urologic Nursing 36: 265-266.

18. Polit D, Beck C (2012) Nursing research: Generating and assessing evidence for nursing practice. 9th ed. Philadelphia, PA: Wolters Kluwer/Lippincott, Williams and Wilkins.

19. Henline SD, Weatherspoon D, Tennison TA (2017) Baby steps to evidence-based practice. Int $J$ Child Birth Educ 32: 26-30.

20. Deng GE, Rausch SM, Jones LW, Gulati A, Kumar NB, et al. (2013) Complementary therapies and integrative medicine in lung cancer: Diagnosis and management of lung cancer. 3rd Ed. American College of Chest Physicians evidence-based clinical practice guidelines. Chest 143: e420S-36S. [Crossref]

Copyright: (C2018 Bonham E. This is an open-access article distributed under the terms of the Creative Commons Attribution License, which permits unrestricted use, distribution, and reproduction in any medium, provided the original author and source are credited. 